ARTICLE

\title{
Flat epitaxial quasi-1D phosphorene chains
}

Wei Zhang ${ }^{1}$, Hanna Enriquez', Yongfeng Tong ${ }^{2}$, Andrew J. Mayne (i) ${ }^{1}$, Azzedine Bendounan², Alex Smogunov³, Yannick J. Dappe ${ }^{3}$, Abdelkader Kara ${ }^{4}$, Gérald Dujardin ${ }^{1} \&$ Hamid Oughaddou (1) 1,5凶

The emergence of peculiar phenomena in 1D phosphorene chains ( $P$ chains) has been proposed in theoretical studies, notably the Stark and Seebeck effects, room temperature magnetism, and topological phase transitions. Attempts so far to fabricate $P$ chains, using the top-down approach starting from a few layers of bulk black phosphorus, have failed to produce reliably precise control of $P$ chains. We show that molecular beam epitaxy gives a controllable bottom-up approach to grow atomically thin, crystalline $1 \mathrm{D}$ flat $\mathrm{P}$ chains on a $\mathrm{Ag}$ (111) substrate. Scanning tunneling microscopy, angle-resolved photoemission spectroscopy, and density functional theory calculations reveal that the armchair-shaped chains are semiconducting with an intrinsic $1.80 \pm 0.20 \mathrm{eV}$ band gap. This could make these $P$ chains an ideal material for opto-electronic devices.

\footnotetext{
${ }^{1}$ Université Paris-Saclay, CNRS, Institut des Sciences Moléculaires d'Orsay, Orsay, France. ${ }^{2}$ TEMPO Beamline, Synchrotron SOLEIL, Gif-sur-Yvette, Cedex France. ${ }^{3}$ Université Paris-Saclay, CNRS, CEA, Service de Physique de l'Etat Condensé, Gif-sur-Yvette, France. ${ }^{4}$ Department of Physics, University of Central

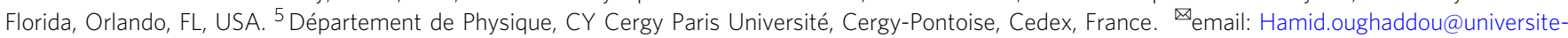
paris-saclay.fr
} 
T wo-dimensional (2D) materials have been at the forefront of research over the past decade. Following the discovery of graphene $e^{1,2}$, many different $2 \mathrm{D}$ materials have appeared since: silicene ${ }^{3,4}$, transition-metal dichalcogenides ${ }^{5}$, germanene $e^{6}$, borophene $e^{7,8}$, and phosphorene ${ }^{9,10}$. While the remarkable properties of these 2D materials are now known, reducing the dimensionality reveals physical properties that help to overcome certain limitations. However, the fabrication of chains based on $2 \mathrm{D}$ materials has proven to be a huge challenge. Many attempts to shape 2D materials into quasi-one-dimensional (quasi-1D) structures have been made, notably in the case of graphene, where lithography and etching techniques were first applied to make nanoribbons $(\mathrm{NRs})^{11}$. Unfortunately, this approach is severely limited by the lack of control over the width, length, and disordered structure of the NRs that could be obtained ${ }^{12}$, preventing any reproducible measurements or implementation into largerscale electronic circuits. This was overcome by the use of a bottom-up chemical synthesis approach that achieves perfect control of the graphene $\mathrm{NR}^{13}$. An energy gap is induced in the narrow graphene NRs that does not otherwise exist in 2D graphene, thus enabling a field-effect transistor to be operated ${ }^{12,14}$.

Among 2D materials, black phosphorus (BP) is composed of a $2 \mathrm{D}$ lattice of $s p^{3}$ hybridized phosphorus atoms arranged in a buckled sheet. BP has several properties that distinguish it from other $2 \mathrm{D}$ materials; in particular, it has a strong in-plane anisotropy ${ }^{15}$ and an intrinsic direct band gap that can be tuned from $1.80 \mathrm{eV}$ for a monolayer (ML) ${ }^{16}$ to $0.35 \mathrm{eV}$ for bulk BP crystals ${ }^{9}$. It is suitable for nanoscale device applications because of a high on/off ratio $\left(10^{5}\right)$ and large carrier mobility $\left(\sim 1000 \mathrm{~cm}^{2} / \mathrm{V} / \mathrm{s}\right)^{9,17,18}$.

There is a large family of phosphorus allotropes, which can be subdivided into different categories. Each category exhibits vastly different crystalline structures and properties. White phosphorus contains four phosphorus atoms arranged in $\mathrm{P}_{4}$ tetrahedrons ${ }^{19}$. Although white phosphorus is one of the most common phosphorus allotropes, it is highly unstable under ambient condition $\mathrm{s}^{20}$. However, red phosphorus is a relatively stable allotrope of phosphorus with a polymeric chain-like structure ${ }^{21}$. Black phosphorus is the most stable allotrope ${ }^{22}$. Since each phosphorus atom has five valence electrons, each atom is bonded to three adjacent phosphorus atoms with $s p^{3}$ hybridized orbitals, resulting in a puckered honeycomb structure ${ }^{23}$, in which the single atomic layers are stacked together by weak van der Waals interactions instead of covalent bonds. BP possesses two distinctive lattice constants for the armchair $(4.37 \AA)$ and zigzag edges $(3.31 \AA)$, respectively. Blue phosphorus, another unique and stable phase of phosphorus, was only grown by the molecular beam epitaxy (MBE) using black phosphorus or InP as precursor ${ }^{24,25}$, presenting a buckled honeycomb structure. This structure presenting zigzag puckering can be obtained by a specific translational dislocation of black phosphorene ${ }^{26}$. Recently, pure violet phosphorus has been reported ${ }^{27}$. It is composed of tubular strands with a monoclinic crystal structure ${ }^{28}$. Fibrous phosphorus also belongs to the family of phosphorus allotropes, composed of $1 \mathrm{D}$ tubular layers held together by van der Waals interactions ${ }^{29,30}$.

Theoretical studies ${ }^{31-40}$ have predicted that reducing the dimensionality of a single atomic layer of phosphorus into $1 \mathrm{D} P$ chains should reveal peculiar phenomena, in particular the Stark effect $^{34}$, the Seebeck effect ${ }^{35}$, room temperature (RT) magnetism ${ }^{36}$, and topological phase transitions ${ }^{37}$.

Top-down methods have been reported for P NRs (PNRs) from bulk black phosphorus ${ }^{41-43}$. Using ionic scissoring of macroscopic black phosphorus crystals, high-quality individual PNRs have been produced $^{41}$. Electro-chemical exfoliation following BP synthesis has been also reported to give phosphorene sheets and NRs ${ }^{42}$. However, both the lithography and electro-chemical techniques are not yet able to produce or control the width of the narrowest PNRs. The controlled fabrication of perfectly defined ribbons has been achieved for graphene by the polymerization of molecular precursors ${ }^{13}$. However, until now this method is still not yet transferable to phosphorene due to its stronger sensitivity to air ${ }^{44}$ and chemical reactivity ${ }^{45,46}$ compared to graphene ${ }^{47}$. In the literature, most studies have looked at 3-10 nm-wide quasi-1D NRs or stripes that can form $2 \mathrm{D}$ arrays.

Here we present the synthesis of self-assembled crystalline phosphorene-like chains on a $\mathrm{Ag}(111)$ surface; the breakthrough is achieved using the MBE procedure to provide precise control. The atomic and electronic structures of $\mathrm{P}$ chains are determined by in situ scanning tunneling microscopy/spectroscopy (STM/ STS) operated at $77 \mathrm{~K}$, low energy electron diffraction (LEED), and high-resolution angular photoemission spectroscopy (HRARPES). Large areas of high-quality $\mathrm{P}$ chains are successfully synthesized, with a measured band gap of $1.8 \mathrm{eV}$. X-ray photoelectron spectroscopy (XPS) analysis reveals a weak interaction between the $\mathrm{P}$ chains and the $\mathrm{Ag}(111)$ substrate, which precludes the existence of any potential silver-phosphorus alloy. The STM topography of the $\mathrm{P}$ chains and the band gap obtained in the spectroscopy are confirmed by density functional theory (DFT) calculations. The atomically precise synthesis of $\mathrm{P}$ chains represents a breakthrough toward the rapid integration of $\mathrm{P}$ chains in real devices by overcoming the limitations of the top-down approach to $\mathrm{P}$ chain synthesis from black phosphorus. The growth is performed in an ultra-high vacuum (UHV) apparatus equipped with the standard tools for growth and characterization (see "Methods").

\section{Results}

Figure 1a presents a large topographic STM image corresponding to low-coverage deposition of $\mathrm{P}$ atoms ( $\sim .1 \mathrm{ML})$ on the $\mathrm{Ag}(111)$ surface. This image shows different atomically flat $\operatorname{Ag}(111)$ terraces, covered by isolated $\mathrm{P}$ chains. Their number depends on the
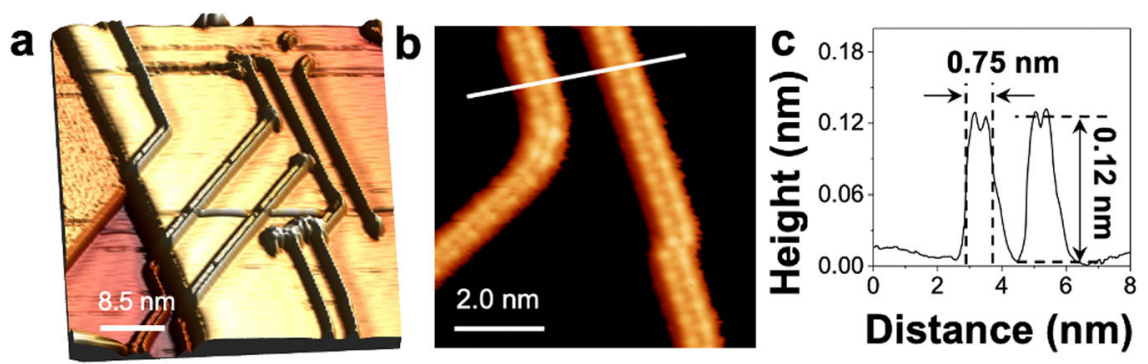

Fig. 1 Formation of $\mathbf{P}$ chains from the early stage of growth. a 3D view of the STM topographic image of $P$ chains on the Ag(111) surface, showing atomically flat terraces and $P$ chains $\left(46 \times 46 \mathrm{~nm}^{2}, U=-800 \mathrm{mV}, I=1.50 \mathrm{nA}\right)$. b High-resolution STM image of $\mathrm{P}$ chains on the Ag(111) $\left(7 \times 7 \mathrm{~nm}{ }^{2}, U=\right.$ $-80 \mathrm{mV}, I=1.50 \mathrm{nA}$ ). $\mathbf{c}$ Line profile measured along white line in $\mathbf{b}$ shows that the $P$ chains have a typical width of $0.75 \pm 0.01 \mathrm{~nm}$ and a height of $0.12 \mathrm{~nm}$. 

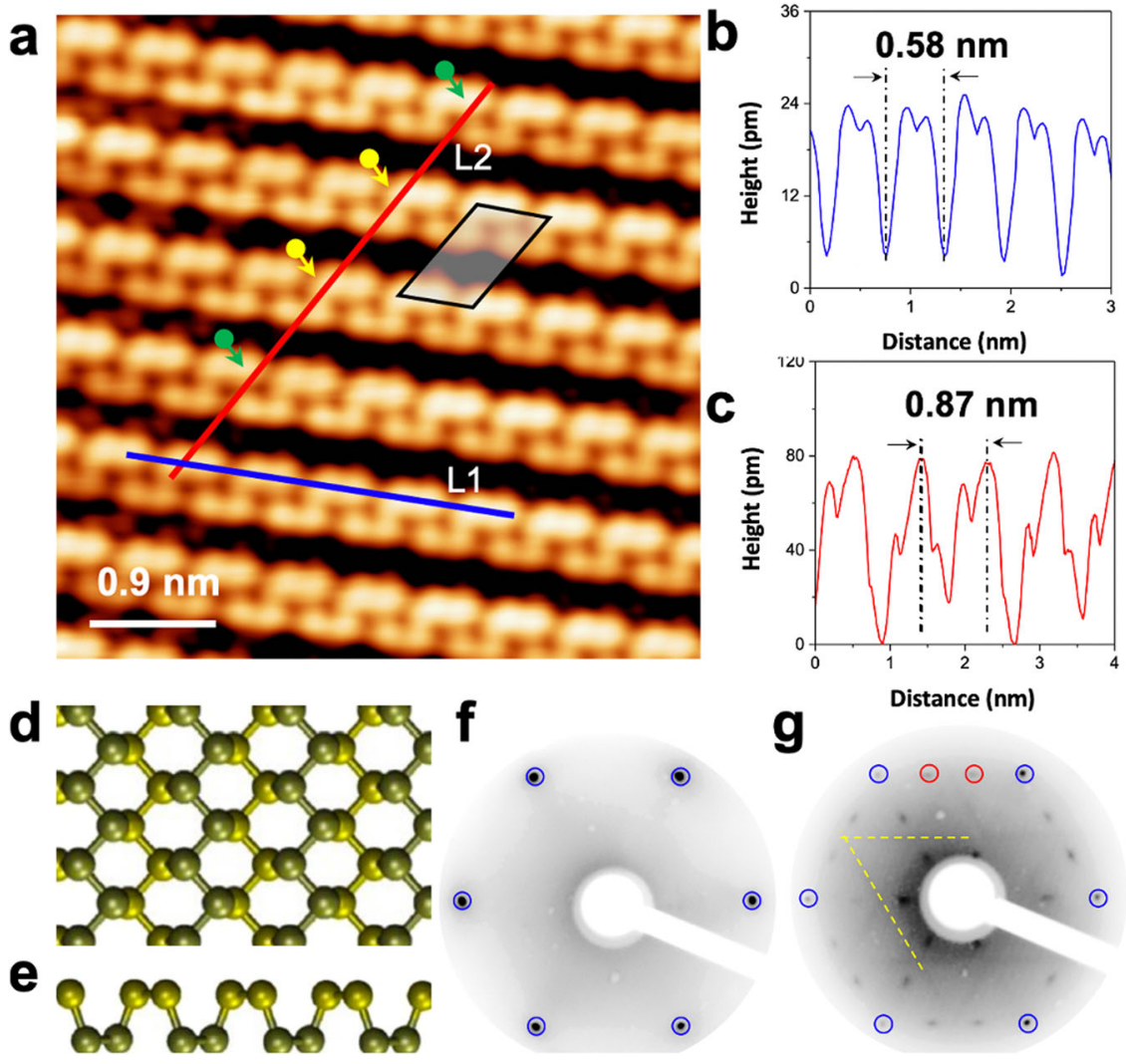

Fig. 2 Atomic structures of $\mathbf{P}$ chains on $\mathbf{A g ( 1 1 1 )}$. a Atomically resolved STM image, showing parallel P chains $\left(5 \times 5 \mathrm{~nm}{ }^{2}, U=-0.1 \mathrm{~V}, I=1.00 \mathrm{nA}\right)$. The unit cell of $\mathrm{P}$ chain structure is indicated by the black rhombus. $\mathbf{b}$ Line scan measured along the line $\mathrm{L} 1 \mathrm{in} \mathbf{a}$. The periodicity of the brightest protrusions is around $0.58 \pm 0.01 \mathrm{~nm}$, corresponding to twice the lattice constant of the $\mathrm{Ag}(111)$ surface $(2 \times 0.289 \mathrm{~nm}=0.578 \mathrm{~nm})$. c Line scan measured along the line L2 in $\mathbf{b}$. The line profile L2 measures the corrugation across the P chains along the other main direction of $\mathrm{Ag}(111)$ in $\mathbf{a}$ and shows a periodicity of $0.87 \pm$ $0.01 \mathrm{~nm}$, which is equal to three times the lattice constant of $\mathrm{Ag}(111)$ surface $(3 \times 0.289 \mathrm{~nm}=0.867 \mathrm{~nm})$. d, e Structural model of black phosphorene layer viewed from the top and side, respectively. $\mathbf{f}, \mathbf{g}$ Low energy electron diffraction patterns recorded at $56 \mathrm{eV}$ corresponding to the bare $\mathrm{Ag}$ (111) and after deposition of $\sim 0.8 \mathrm{ML}$ of $\mathrm{P}$ on $\mathrm{Ag}(111)$, respectively. The $(1 \times 1)$ spots of $\mathrm{Ag}$ substrate are highlighted by blue circles, the $\times 3$ are highlighted by red circles, and the $\times 2$ are highlighted by dashed yellow lines.

P coverage; at low coverage, only a few chains are observed, while at higher coverage, the $\mathrm{P}$ chains assemble into $2 \mathrm{D}$ arrays (Fig. 2a). A number of examples in the literature support this coveragedependent behavior: silicene on $\mathrm{Ag}(110)^{48}$, silicene on $\mathrm{Au}$ $(110)^{49,50}$, and $\mathrm{Si}$ on $\mathrm{SiC}(001)^{51}$. We observe that, at the earliest stage of growth, $\mathrm{P}$ atoms are not disordered but the $\mathrm{P}$ chains are formed rapidly. Figure $1 \mathrm{~b}$ (zoom of Fig. 1a) presents an atomically resolved STM image of individual P chains. The $\mathrm{P}$ chains have the same internal atomic structure, consisting of two rows of bright protrusions, with a width of $0.75 \pm 0.01 \mathrm{~nm}$ and a height of $0.120 \pm 0.001 \mathrm{~nm}$ (line profile in Fig. 1c). The $\mathrm{P}$ chains lie on top of the $\operatorname{Ag}(111)$ surface and are aligned along the main crystallographic directions; a few kinks and bends are visible.

After deposition of $\sim 0.8 \mathrm{ML}$ of phosphorus on $\mathrm{Ag}(111)$, a high density of self-assembled $\mathrm{P}$ chains with different domains is visible in the large-scale STM image (Supplementary Fig. 1a). The lengths of the P chains were analyzed from the STM images taken at random positions on the surface. Supplementary Fig. 1b shows a histogram of the distribution of the chain lengths. Despite the finite image size that truncates the longer $\mathrm{P}$ chains ${ }^{52}$, their average length is around $25 \mathrm{~nm}$. The fine structural details in the atomicresolved STM image in Fig. 2a reveals an armchair-like structure of the $\mathrm{P}$ chains. The distance between the first nearest-neighbor protrusions is $0.21 \pm 0.01 \mathrm{~nm}$, close to that of $\mathrm{BP}$, while the corrugation between neighboring $\mathrm{P}$ atoms is very small $(<10 \mathrm{pm})$, indicating that all the atoms have the same height with respect to the surface plane. The line profile of Fig. 1c shows that the P chains have the height of a single layer.

In that respect, the interaction with the $\operatorname{Ag}(111)$ surface causes the natural buckled chain structure to be flattened in the armchair direction. The dimensions of the unit cell are $0.58 \mathrm{~nm} \times 0.87 \mathrm{~nm}$ from the line profiles L1 and L2 (Fig. 2b, c, respectively). The unit cell dimensions and line profiles indicate that the $\mathrm{P}$ chains selfassemble to form a $(2 \times 3)$ superstructure relative to the $\operatorname{Ag}(111)$ surface. Note that the $2 \times 3$ periodicity of $\mathrm{P}$ chains is not perfect because some $\mathrm{P}$ chains are shifted laterally relative to the neighboring nanowires by one Ag lattice parameter as indicated in Fig. 2a by the green and yellow arrows, respectively.

A comparison of the atomic-resolved STM image resembles remarkably the ball and stick model (Fig. 2d) of a black phosphorene layer viewed from the side (Fig. 2e), suggesting that the obtained structure corresponds to flat $\mathrm{P}$ chains with an armchair structure. If we consider the projection of the side view (Fig. 2e) of the black phosphorene, it has the armchair structure (two up atoms and two down atoms) due to the puckered arrangement of the $\mathrm{P}$ atoms. In this "phosphorene-like" form, these armchair chains of phosphorene atoms lie in the plane parallel to the surface. Hence, this observed form of phosphorene is flat.

The surface was also characterized using LEED. The LEED patterns were recorded at $56 \mathrm{eV}$, on the bare $\mathrm{Ag}(111)$ surface (Fig. 2f) and after deposition of $\sim 0.8 \mathrm{ML}$ of $\mathrm{P}$ (Fig. $2 \mathrm{~g}$ ). In both images, the diffraction spots corresponding to the $\operatorname{Ag}(111)$ 

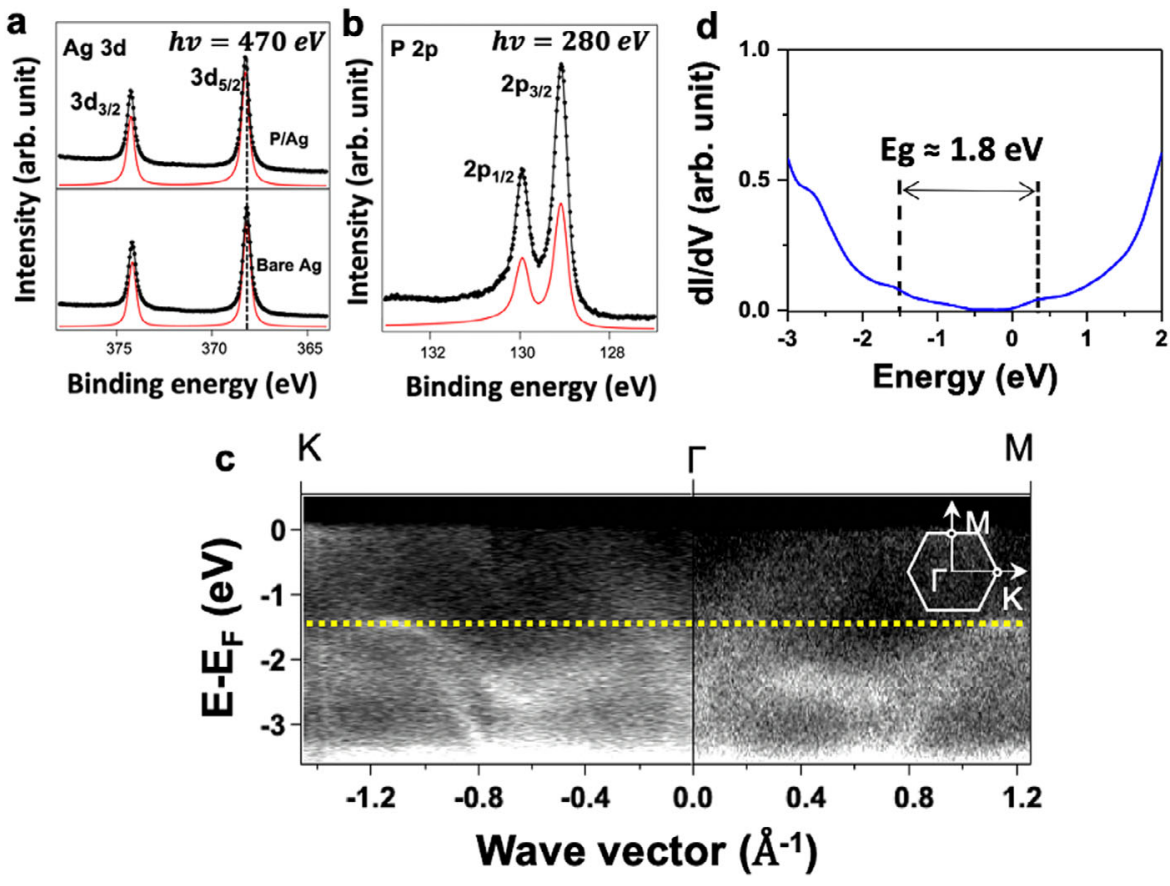

Fig. 3 Experimental XPS, HR-ARPES, and simulated spectra of $\mathbf{P}$ chains on Ag(111). a XPS spectra of Ag $3 d$ core levels before (lower panel) and after growth of $\mathrm{P}$ chains (upper panel). b $\mathrm{P} 2 p$ core-level spectrum, presenting only one doublet (red curve). (Black dots present the data; black curve presents the best fit.) c The HR-ARPES were recorded at photon energy of $60 \mathrm{eV}$ along $K-\Gamma-M$ directions after deposition of $P$ chains on $A g(111)$ surface. $\mathbf{d}$ STS $\mathrm{d} l / \mathrm{dV}$ spectra acquired on the $\mathrm{P}$ chains, demonstrating the semiconducting character of $\mathrm{P}$ chains. The curve is an average of 40 individual spectra.

surface are indicated by blue circles. Following the deposition of $\mathrm{P}$ atoms, different spots appear corresponding to a $\times 3$ structure (red circles) with respect to the silver substrate in good agreement with the STM observations. The elongated spots of the $\times 3$ structure indicate that the order is not perfect along this direction. This is due to the shift between two neighboring $\mathrm{P}$ chains that we see in the STM images (Fig. 2a). The thin elongated streaks with $\mathrm{a} \times 2$ periodicity appear weak in the diffraction pattern (outlined by the yellow dashed line). This weakness is due to an inter $\mathrm{P}$ chain shift of one atomic parameter of silver. This behavior was also observed in the case of silicene NRs on $\operatorname{Ag}(110)^{50}$.

The chemical environment at the interface between the asgrown $\mathrm{P}$ chains and the substrate was characterized by XPS measurements. Figure $3 \mathrm{a}$ shows the $\mathrm{Ag} 3 d$ core-level spectra corresponding to the bare substrate and after deposition of $\sim 0.8$ $\mathrm{ML}$ of $\mathrm{P}$ atoms, respectively. For both spectra, the characteristic $\operatorname{Ag} 3 d_{3 / 2}$ and $3 d_{5 / 2}$ peaks are located at the same binding energies of 374.2 and $368.2 \mathrm{eV}$, respectively (see "Methods"). The binding energy values and their shapes remain unchanged. We also observe no detectable peak splitting nor any significant energy shifts after the phosphorus deposition, pointing to a weak interaction between the $\mathrm{P}$ chains and the Ag surface. The P $2 p$ corelevel spectrum presented in Fig. $3 \mathrm{~b}$ reveals only one spin-orbit doublet indicating that $\mathrm{P}$ atoms have only one chemical environment. The $2 p_{1 / 2}$ and $2 p_{3 / 2}$ peaks are located at 129.89 and $129.03 \mathrm{eV}$, respectively. In addition, no peak is detected at higher binding energy, which would correspond to oxidized phosphorus ${ }^{53}$. This indicates that the as-grown $\mathrm{P}$ chains on the $\mathrm{Ag}(111)$ are composed of pure phosphorus with a high structural quality. The STM and XPS results concord that the P chains are flat on the surface with an armchair-like structure in which the $\mathrm{P}$ atoms have the same chemical environment.

The electronic structure of the $\mathrm{P}$ chains is also explored through a combination of HR-ARPES and STS measurements. Figure $3 \mathrm{c}$ shows the band structure of the $\mathrm{P}$ chains obtained by HR-ARPES. Measurements were recorded parallel to and perpendicular to the armchair direction. In reciprocal space, this corresponds to the $\Gamma-K$ and $\Gamma-M$ directions, respectively. A dispersive band associated with the phosphorene layer is located at $1.50 \pm 0.20 \mathrm{eV}$ below the Fermi level, indicating unambiguously the existence of an energy gap of at least $1.5 \mathrm{eV}$. Despite the 1D character of the $\mathrm{P}$ chains, we measure here the electronic band structure associated with the $2 \mathrm{D}$ network of $\mathrm{P}$ chains adsorbed on the $\operatorname{Ag}(111)$ surface. This explains the observation of electronic bands in the direction perpendicular to the chains $(\Gamma-K)$. This type of peculiar band was observed on an exfoliated single layer of black phosphorus and was explained by the contribution from a bonding and anti-bonding pair of the $\mathrm{P} 2 p_{z}$ orbitals $^{54}$. These bands are observed here on an epitaxial phosphorene ML and there is almost no influence of the underlying Ag substrate, which reflects a very weak interaction at the interface.

Figure $3 \mathrm{~d}$ shows a typical STS $\mathrm{d} I / \mathrm{d} V$ spectrum recorded on the $P$ chains presenting a clear semiconducting character. At negative bias, the local density of states (LDOS) is flat and featureless between the Fermi level and $U_{\text {gap }}=-1.5 \mathrm{~V}$. The $s p$ state of the underlying silver contributes a slight rise in the DOS between -0.7 and $-1.5 \mathrm{~V}$ but is strongly attenuated by the $\mathrm{P}$ chains. This behavior is seen in the case of $\mathrm{NaCl} / \mathrm{Ag}^{55,56}$, and as we describe below, the band folding within the $2 \times 3$ unit cell induces a background in the spectra. The peak located at $-1.5 \mathrm{~V}$ is attributed to the valence band maximum, consistent with the HRARPES observation (horizontal yellow dashed line in Fig. 3c). At positive bias, the conduction band minimum is located at $U_{\text {gap }}=$ $+0.3 \mathrm{~V}$ above the Fermi level. Combining the STS and HRARPES gives a band gap close to $1.80 \pm 0.20 \mathrm{eV}$ for the $\mathrm{P}$ chains.

To confirm the experimental observations, we performed DFT calculations (see "Methods"). After full relaxation of the system, the resulting structure is composed of an ordered array of phosphorene chains (Fig. 4a, b), closely resembling the STM observations (Fig. 2a). We find a binding energy of $0.71 \mathrm{eV}$ per $\mathrm{P}$ atom, reflecting an interaction that can be described as either weak chemisorption or a strong physisorption. The calculated 

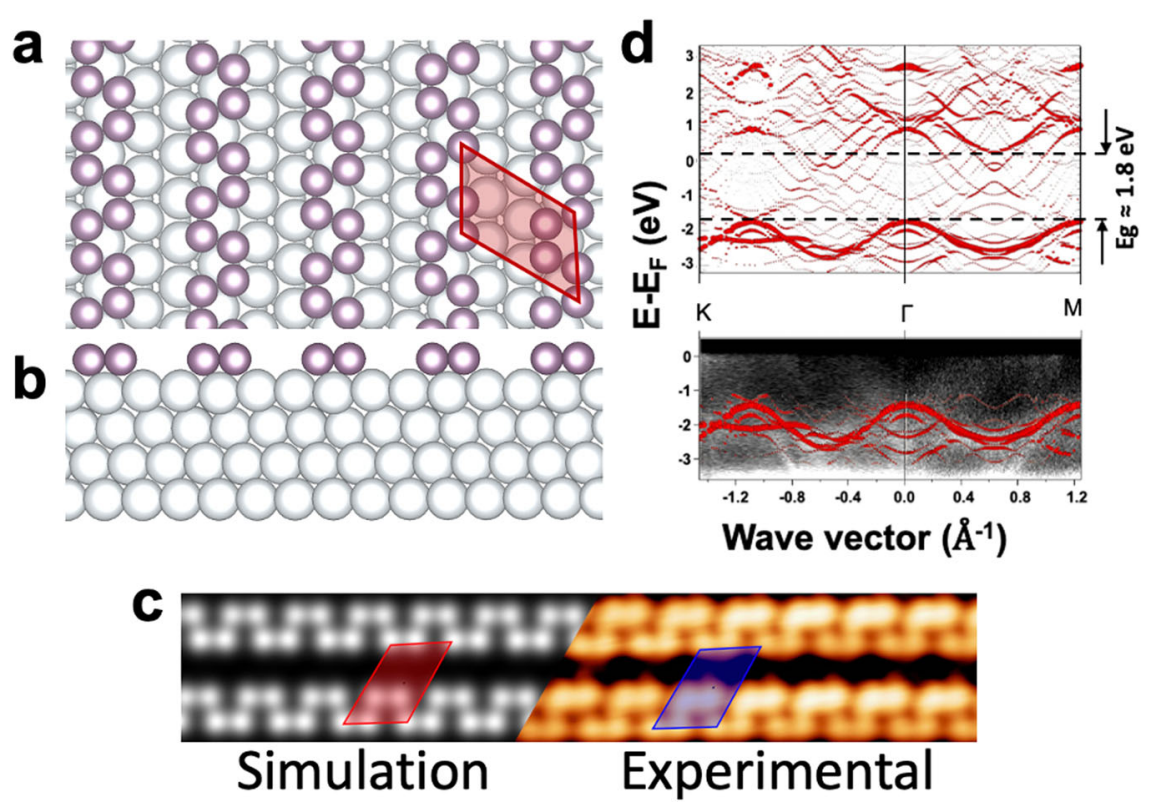

Fig. 4 Model of PNR structure from DFT calculations. a Top view showing the armchair PNR structure. P atoms are in purple, Ag atoms in gray. The $2 \times 3$ unit cell is indicated by the red parallelepiped. $\mathbf{b}$ Side view showing the atoms of the P chains lying flat on the Ag substrate. c Simulated (left) and experimental (right) STM images. d (upper panel) The calculated DFT band structure of the whole $4 \mathrm{P} /(2 \times 3)-\mathrm{Ag}(111)$ surface along the $K-\Gamma-M$ path of the $(1 \times 1)-A g(111)$ surface using $160 \mathrm{k}$-points. $\mathbf{d}$ (lower panel) The superposition of the experimental and the calculated ARPES shows an excellent agreement.

corrugation of $\mathrm{P}$ chains is negligible $(0.004 \mathrm{~nm})$ and in excellent agreement with the STM results. The calculated nearest-neighbor distance in the $\mathrm{P}$ chains was found to be $0.22 \mathrm{~nm}$ in close agreement with observation $(0.21 \mathrm{~nm})$. We simulated the STM image from the partial charge density of the relaxed systems using the Tersoff-Hamann approach (see "Methods"). Figure 4c shows side by side the experimentally determined image (right) and the computed image (left). The calculated STM image from the proposed model is in excellent agreement with the experimental STM image.

To complement this, we also calculated the electronic band structure of the system (Fig. 4d). We present here the contribution of $\mathrm{P}$ chains of the full $\mathrm{P} / \mathrm{Ag}(111)$ system but the contribution of $\mathrm{P}$ and $\mathrm{Ag}$ cannot be decoupled experimentally. The contribution of the $\mathrm{P}$ atoms is superimposed on the experimental HRARPES. The results fit the experimentally observed dispersion band very well (Fig. 3c). Obviously using $k$-points from the first Brillouin zone of the $(1 \times 1)-\operatorname{Ag}(111)$ unit cell induces the appearance of several folded bands. However, one can observe the standard parabolic dispersion of silver around the $\Gamma$ point. In addition, the $\mathrm{P}$ contribution at $1.60 \pm 0.20 \mathrm{eV}$ below the Fermi level is clearly visible and is close to the experimental value (1.5 $\mathrm{eV}$ ). From the calculations, the band gap equals $1.80 \mathrm{eV}$ in good agreement with STS measurements.

Further calculations and analysis are presented in the Supplementary Information, in particular the calculated band structure of an isolated $\mathrm{P}$ chain without the Ag substrate exhibiting a metallic character (Supplementary Fig. 2). In comparison, Supplementary Fig. 3a, b shows the band structure and the DOS of a hydrogenated $\mathrm{P}$ chain, respectively. The hydrogenation leads to the opening of a band gap in the electronic structure. These calculations support the fact that the Ag substrate passivates the $\mathrm{P}$ chains. Interestingly, the band gap is indirect on Ag, compared to $\mathrm{H}$-passivated free-standing phosphorene, which might be attributed to variations in the local charge transfer. Moreover, we have probed the interaction between the $\mathrm{P}$ chains in the bidimensional array by calculating the DOS of the isolated $\mathrm{P}$ chain (in a large Ag unit cell) compared to the chain in the array
(Supplementary Fig. 4). The DOS from these two calculations are very similar, which indicates that there is no direct interaction between the chains, rather an indirect one through the $\mathrm{Ag}$ substrate.

The band structure of the bare $2 \times 3-\operatorname{Ag}(111)$ unit cell has also been calculated (Supplementary Fig. 5), showing in conjunction with Supplementary Fig. 2 that the full band structure does not result in the simple superposition of the band structures of the isolated systems. Besides, it is rather difficult to interpret the features above the yellow line in Fig. 3d because of the band folding of the silver surface, which gives rise to an electronic background covering all this specific area of the Brillouin zone. In addition, spin-polarized calculations have been performed showing no magnetism in the P chain (Supplementary Fig. 8). Finally, ab initio molecular dynamics simulations have been performed at $500 \mathrm{~K}$ to probe the stability of the $\mathrm{P}$ chains on the Ag surface. The results shown in Supplementary Figs. 9 and 10 reveal a strong stability of the system in agreement with the experiments.

In summary, we present a controlled synthesis of flat phosphorene-like $\mathrm{P}$ chains on $\mathrm{Ag}(111)$ using the MBE process. The atomic and electronic structures of these epitaxial P chains were experimentally determined and confirmed by DFT calculations. The $\mathrm{P}$ chains self-assemble on $\mathrm{Ag}(111)$ surface and form an armchair-like structure giving a $(2 \times 3)$ superstructure. They are semiconducting with a band gap of $1.8 \mathrm{eV}$. The discovery of $\mathrm{P}$ chains on $\mathrm{Ag}(111)$ using the bottom-up approach opens the way for rapid technological advances based on phosphorene. Our contribution concerns a first step along this road, namely, the fabrication of well-defined 1D P chains by MBE methods, which in itself constitutes a breakthrough. Our STM study shows that the 1D P chains have a uniform armchair structure and are semiconducting. Nevertheless, realistically several challenges remain; the first is the non-negligible interaction of the $1 \mathrm{D} P$ chains with the Ag substrate. This can be overcome by hydrogenation of the $\mathrm{P}$ chains ${ }^{39,57}$ or growth on a thin $\mathrm{NaCl}$ layer ${ }^{56}$. In these two examples, one can anticipate the $\mathrm{P}$ chains to show a direct electronic band gap. This would enable the excitonic 
structure of the $\mathrm{P}$ chains to be investigated by STM luminescence as has been demonstrated recently on $\mathrm{MoSe}_{2}$ flakes ${ }^{58}$.

\section{Methods}

Experiments. The experiments were carried out with a commercial UHV system, consisting of three inter-connected chambers, where the base pressure is better than $3.0 \times 10^{-10}$ mbar. The single-crystal $\operatorname{Ag}(111)$ substrate (99.999\%) was cleaned by several cycles of $\mathrm{Ar}^{+}$sputtering and post-annealing. The beam energy is $2 \mathrm{keV}$ at $5 \times 10^{-6}$ mbar and the subsequent annealing temperature of $500^{\circ} \mathrm{C}$ is maintained for $40 \mathrm{~min}$ to remove any contamination. A Knudsen cell loaded with commercial bulk black phosphorus was used as a phosphorus source to grow the phosphorene chains. A series of experiments was performed with various phosphorus coverages corresponding to deposition times ranging from 1 to $8 \mathrm{~min}$, in which high-purity phosphorus was deposited onto $\operatorname{Ag}(111)$ while the substrate was kept at RT. During growth, the Knudsen cell was heated to $450{ }^{\circ} \mathrm{C}$ giving a stable deposition rate of $0.1 \mathrm{ML} / \mathrm{min}$, calibrated by a quartz microbalance. The surface structure and chemical composition were monitored by LEED and AES measurements performed at RT. The morphology of sample was characterized by in situ low-temperature STM, operated at 77 K. All STM measurements were recorded at a constant current mode. The tungsten (W) tips were made by electrochemical etching. STS experiments were measured at $77 \mathrm{~K}$ using a standard lock-in technique, where a modulation voltage of $10 \mathrm{mV}$ at a frequency of 5127.7 $\mathrm{Hz}$ was applied.

The photoemission experiments were performed using the same substrate on the TEMPO beamline of the Synchrotron SOLEIL-France. The photon energies of the XPS experiments were recorded at $470 \pm 0.20 \mathrm{eV}$ for $\mathrm{Ag} 3 d$ and $280 \pm 0.20 \mathrm{eV}$ for P $2 p$. All spectra are fitted with a Doniach-Sunjic line shape ${ }^{59}$. The best fit of Ag $3 d$ was obtained with a $140 \mathrm{meV}$ Gaussian profile and a $280 \mathrm{meV}$ Lorentzian profile, while the spin-orbit splitting is $6 \mathrm{eV}$. The best fit of P $2 p$ was obtained with a $200 \mathrm{meV}$ Gaussian profile and a $80 \mathrm{meV}$ Lorentzian profile, while the spin-orbit splitting is $0.865 \mathrm{eV}$.

Calculations. The present calculations were performed using DFT. In the calculations, we considered a $2 \times 3-\mathrm{Ag}(111)$ slab of 5 layers on top of which 4 phosphorous atoms were positioned according to the standard geometry of the side view of a black phosphorus layer. To test the size effect, a calculation using nine layers was performed. Correlation effects were treated using the Perdew-Burke-Ernzerhof exchange-correlation functional ${ }^{60}$ as implemented in VASP ${ }^{61-63}$ version 5.4.4. The interaction between the valence electrons and ionic cores was described by the projector augmented wave method ${ }^{64,65}$. The plane wave energy cut-off was set to $400 \mathrm{eV}$. We then optimized the geometry until the residual forces fell $<0.01 \mathrm{eV} / \AA$. The calculated STM images were produced using the Tersoff-Hamann method ${ }^{66}$ and the p4vasp software package with an STM tip placed $2.05 \AA$ above the position of a phosphorus atom.

\section{Data availability}

All data needed to evaluate the conclusions of this study are available in the main text or Supplementary Materials. The data that support the findings of this study are available from the corresponding authors upon reasonable request.

Received: 23 October 2020; Accepted: 21 July 2021; Published online: 27 August 2021

\section{References}

1. Novoselov, K. S. et al. Two-dimensional gas of massless Dirac fermions in graphene. Nature 438, 197-200 (2005).

2. Zhang, Y., Tan, Y.-W., Stormer, H. L. \& Kim, P. Experimental observation of the quantum Hall effect and Berry's phase in graphene. Nature 438, 201-204 (2005).

3. Lalmi, B. et al. Epitaxial growth of a silicene sheet. Appl. Phys. Lett. 97, 223109 (2010).

4. Tao, L. et al. Silicene field-effect transistors operating at room temperature. Nat. Nanotechnol. 10, 227-231 (2015).

5. Manzeli, S., Ovchinnikov, D., Pasquier, D., Yazyev, O. V. \& Kis, A. 2D transition metal dichalcogenides. Nat. Rev. Mater. 2, 17033 (2017).

6. Derivaz, M. et al. Continuous germanene layer on $\mathrm{Al}(111)$. Nano Lett. 15, 2510-2516 (2015).

7. Mannix, A. J. et al. Synthesis of borophenes: anisotropic, two-dimensional boron polymorphs. Science 350, 1513-1516 (2015).

8. Feng, B. et al. Experimental realization of two-dimensional boron sheets. Nat. Chem. 8, 563-568 (2016).

9. Li, L. et al. Black phosphorus field-effect transistors. Nat. Nanotechnol. 9, 372-377 (2014).
10. Liu, H. et al. Phosphorene: an unexplored 2D semiconductor with a high hole mobility. ACS Nano 8, 4033-4041 (2014)

11. Tapasztó, L., Dobrik, G., Lambin, P. \& Biro, L. P. Tailoring the atomic structure of graphene nanoribbons by scanning tunnelling microscope lithography. Nat. Nanotechnol. 3, 397-401 (2008).

12. Jiao, L., Zhang, L., Wang, X., Diankov, G. \& Dai, H. Narrow graphene nanoribbons from carbon nanotubes. Nature 458, 877-880 (2009).

13. Cai, J. et al. Atomically precise bottom-up fabrication of graphene nanoribbons. Nature 466, 470-473 (2010).

14. Li, X., Wang, X., Zhang, L., Lee, S. \& Dai, H. Chemically derived, ultrasmooth graphene nanoribbon semiconductors. Science 319, 1229-1232 (2008).

15. Wang, X. et al. Highly anisotropic and robust excitons in monolayer black phosphorus. Nat. Nanotechnol. 10, 517-521 (2015).

16. Li, L. et al. Direct observation of the layer-dependent electronic structure in phosphorene. Nat. Nanotechnol. 12, 21-25 (2017).

17. Qiao, J., Kong, X., Hu, Z.-X., Yang, F. \& Ji, W. High-mobility transport anisotropy and linear dichroism in few-layer black phosphorus. Nat. Commun. 5, 4475 (2014).

18. Xia, F., Wang, H. \& Jia, Y. Rediscovering black phosphorus as an anisotropic layered material for optoelectronics and electronics. Nat. Commun. 5, 4458 (2014).

19. Corbridge, D. \& Lowe, E. Structure of white phosphorus: single crystal X-ray examination. Nature 170, 629-629 (1952).

20. Peruzzini, M., Gonsalvi, L. \& Romerosa, A. Coordination chemistry and functionalization of white phosphorus via transition metal complexes. Chem. Soc. Rev. 34, 1038-1047 (2005).

21. Zhang, S. et al. Towards unveiling the exact molecular structure of amorphous red phosphorus by single-molecule studies. Angew. Chem. Int. Ed. 58, 1659-1663 (2019)

22. Jamieson, J. C. Crystal structures adopted by black phosphorus at high pressures. Science 139, 1291-1292 (1963).

23. Rodin, A. S., Carvalho, A. \& Castro Neto, A. H. Strain-induced gap modification in black phosphorus. Phys. Rev. Lett. 112, 176801 (2014).

24. $\mathrm{Xu}$, J.-P. et al. One-dimensional phosphorus chain and two-dimensional blue phosphorene grown on $\mathrm{Au}(111)$ by molecular-beam epitaxy. Phys. Rev. Mater. 1, 061002 (2017).

25. Zhang, W. et al. Epitaxial synthesis of blue phosphorene. Small 14, 1804066 (2018).

26. Zhu, Z. \& Tománek, D. Semiconducting layered blue phosphorus: a computational study. Phys. Rev. Lett. 112, 176802 (2014).

27. Zhang, L. et al. Structure and properties of violet phosphorus and its phosphorene exfoliation. Angew. Chem. 132, 1090-1096 (2020).

28. Thurn, H. \& Kerbs, H. Crystal structure of violet phosphorus. Angew. Chem Int. Ed. 5, 1047-1048 (1966).

29. Amaral, P. E. et al. Fibrous phosphorus quantum dots for cell imaging. ACS Appl. Nano Mater. 3, 752-759 (2020).

30. Ruck, M. et al. Fibrous red phosphorus. Angew. Chem. Int. Ed. 44, 7616-7619 (2005).

31. Carvalho, A., Rodin, A. \& Neto, A. C. Phosphorene nanoribbons. Europhys. Lett. 108, 47005 (2014).

32. Sorkin, V., Cai, Y., Ong, Z., Zhang, G. \& Zhang, Y.-W. Recent advances in the study of phosphorene and its nanostructures. Crit. Rev. Solid State Mater. Sci. 42, 1-82 (2017).

33. Nourbakhsh, Z. \& Asgari, R. Excitons and optical spectra of phosphorene nanoribbons. Phys. Rev. B 94, 035437 (2016).

34. $\mathrm{Wu}, \mathrm{Q}$. et al. Electronic and transport properties of phosphorene nanoribbons. Phys. Rev. B 92, 035436 (2015).

35. Yang, Y.-R., Zhang, Z.-Q., Gu, L. \& Fu, H.-H. Spin-dependent Seebeck effect in zigzag black phosphorene nanoribbons. RSC Adv. 6, 44019-44023 (2016).

36. Yang, G., Xu, S., Zhang, W., Ma, T. \& Wu, C. Room-temperature magnetism on the zigzag edges of phosphorene nanoribbons. Phys. Rev. B 94, 075106 (2016).

37. Sisakht, E. T., Fazileh, F., Zare, M., Zarenia, M. \& Peeters, F. Strain-induced topological phase transition in phosphorene and in phosphorene nanoribbons. Phys. Rev. B 94, 085417 (2016).

38. Poljak, M. \& Suligoj, T. Immunity of electronic and transport properties of phosphorene nanoribbons to edge defects. Nano Res. 9, 1723-1734 (2016).

39. Zhang, J. et al. Phosphorene nanoribbon as a promising candidate for thermoelectric applications. Sci. Rep. 4, 6452 (2014).

40. Farooq, M. U., Hashmi, A. \& Hong, J. Manipulation of magnetic state in armchair black phosphorene nanoribbon by charge doping. ACS Appl. Mater. Interfaces 7, 14423-14430 (2015).

41. Watts, M. C. et al. Production of phosphorene nanoribbons. Nature 568, 216-220 (2019).

42. Liu, Z. et al. Unzipping of black phosphorus to form zig-zag phosphorene nanobelts. Nat. Commun. 11, 3917 (2020).

43. Feng, X. et al. High mobility anisotropic black phosphorus nanoribbon fieldeffect transistor. Adv. Funct. Mater. 28, 1801524 (2018). 
44. Wang, G., Slough, W. J., Pandey, R. \& Karna, S. P. Degradation of phosphorene in air: understanding at atomic level. 2D Mater. 3, 025011 (2016).

45. Castellanos-Gomez, A. et al. Isolation and characterization of few-layer black phosphorus. 2D Mater. 1, 025001 (2014)

46. Wood, J. D. et al. Effective passivation of exfoliated black phosphorus transistors against ambient degradation. Nano Lett. 14, 6964-6970 (2014).

47. Novoselov, K. S. \& Geim, A. The rise of graphene. Nat. Mater. 6, 183-191 (2007).

48. Aufray, B. et al. Graphene_like silicon nanoribbons on $\mathrm{Ag}(110)$ : a possible formation of silicene. Appl. Phys. Lett. 96, 183102 (2010).

49. Tchalala, M. R. et al. Formation of one-dimensional self-assembled silicon nanoribbons on Au(110)-2x1. Appl. Phys. Lett. 102, 083107 (2013).

50. Tchalala, M. R. et al. Atomic structure of silicene nanoribbons on $\operatorname{Ag}(110)$. J. Phys. Conf. Ser. 491, 012002 (2014).

51. Soukiassian, P., Semond, F., Mayne, A. \& Dujardin, G. Highly stable Si atomic line formation on the b-SiC(100) surface. Phys. Rev. Lett. 79, 2498-2501 (1997).

52. Mayne, A. J., Goringe, C. M., Smith, C. W. \& Briggs, G. A. D. Statistical analysis of adsorbates on semiconductors. Surf. Sci. 348, 209-225 (1996).

53. Edmonds, M. T. et al. Creating a stable oxide at the surface of black phosphorus. ACS Appl. Mater. Interfaces 7, 14557-14562 (2015).

54. Kim, J. et al. Observation of tunable band gap and anisotropic Dirac semimetal state in black phosphorus. Science 349, 723-726 (2015).

55. Olsson, F. E., Paavilainen, S., Persson, M., Repp, J. \& Meyer, G. Multiple charge states of $\mathrm{Ag}$ atoms on ultrathin $\mathrm{NaCl}$ films. Phys. Rev. Lett. 98, 176803 (2007).

56. Quertite, K. et al. Silicene nanoribbons on an insulating thin film. Adv. Funct. Mater. 30, 2007013 (2020).

57. Maibam, A., Chakraborty, D., Joshi, K. \& Krishnamurty, S. Exploring edge functionalised blue phosphorene nanoribbons as novel photocatalysts for water splitting. N. J. Chem. 45, 3570-3580 (2021).

58. Pommier, D. et al. Scanning tunneling microscope-induced excitonic luminescence of a two-dimensional semiconductor. Phys. Rev. Lett. 123, 027402 (2019)

59. Doniach, S. \& Sunjic, M. Many-electron singularity in X-ray photoemission and X-ray line spectra from metals. J. Phys. C Solid State Phys. 3, 285-291 (1970).

60. Perdew, J. P., Burke, K. \& Ernzerhof, M. Generalized gradient approximation made simple. Phys. Rev. Lett. 77, 3865-3868 (1996).

61. Kresse, G. \& Furthmüller, J. Efficient iterative schemes for ab initio totalenergy calculations using a plane-wave basis set. Phys. Rev. B 54, 11169-11186 (1996).

62. Kresse, G. \& Furthmüller, J. Efficiency of ab-initio total energy calculations for metals and semiconductors using a plane-wave basis set. Comput. Mater. Sci. 6, 15-50 (1996).

63. Kresse, G. \& Hafner, J. Ab initio molecular dynamics for liquid metals. Phys. Rev. B 47, 558-561 (1993).

64. Blöchl, P. E. Projector augmented-wave method. Phys. Rev. B 50, 17953-17979 (1994).

65. Kresse, G. \& Joubert, D. From ultrasoft pseudopotentials to the projector augmented-wave method. Phys. Rev. B 59, 1758-1775 (1999).
66. Tersoff, J. \& Hamann, D. Theory and application for the scanning tunneling microscope. Phys. Rev. Lett. 50, 1998-2001 (1983).

\section{Acknowledgements}

W.Z. would like to thank the Chinese Scholarship Council (CSC) for the PhD financia support (scholarship). A.K. acknowledge partial support from the US Department of Energy Basic Energy Science under Contract No. DE-FG02-11ER16243. This research used resources of the National Energy Research Scientific Computing Center (NERSC), which is supported by the Office of Science of the U.S. Department of Energy.

\section{Author contributions}

H.O. conceived and conducted the research project. W.Z., H.E., Y.T., A.B. and H.O performed the experiments. Y.J.D., A.S. and A.K. performed the calculations. All authors analyzed the results and contributed to the scientific discussions and manuscript preparation.

\section{Competing interests}

The authors declare no competing interests.

\section{Additional information}

Supplementary information The online version contains supplementary material available at https://doi.org/10.1038/s41467-021-25262-7.

Correspondence and requests for materials should be addressed to H.O.

Peer review information Nature Communications thanks Noriaki Takagi and the other anonymous reviewers for their contribution to the peer review of this work.

Reprints and permission information is available at http://www.nature.com/reprints

Publisher's note Springer Nature remains neutral with regard to jurisdictional claims in published maps and institutional affiliations.

Open Access This article is licensed under a Creative Commons Attribution 4.0 International License, which permits use, sharing, adaptation, distribution and reproduction in any medium or format, as long as you give appropriate credit to the original author(s) and the source, provide a link to the Creative Commons license, and indicate if changes were made. The images or other third party material in this article are included in the article's Creative Commons license, unless indicated otherwise in a credit line to the material. If material is not included in the article's Creative Commons license and your intended use is not permitted by statutory regulation or exceeds the permitted use, you will need to obtain permission directly from the copyright holder. To view a copy of this license, visit http://creativecommons.org/ licenses/by/4.0/.

(C) The Author(s) 2021 